\title{
Correction to: Characterizing natural riparian vegetation for modeling of flow and suspended sediment transport
}

\author{
Kaisa Västilä ${ }^{1}$ Juha Järvelä ${ }^{1}$
}

Published online: 4 November 2017

(C) Springer-Verlag GmbH Germany 2017

Erratum to: J Soils Sediments

https://doi.org/10.1007/s11368-017-1776-3

The authors regret that the article contains a typing mistake in Eq. (5), with an $u_{C}{ }^{2}$ term accidentally appearing at the right hand side of the squared brackets. Originally, Eq. (5) was correctly derived in Västilä and Järvelä (2014) and should read:

$f^{\prime \prime}=4\left[C_{D \chi, F}\left(\frac{u_{C}}{u_{\chi, F}}\right)^{\chi_{F}} \frac{A_{L}}{A_{B}}+C_{D \chi, S}\left(\frac{u_{C}}{u_{\chi, S}}\right)^{\chi_{S}} \frac{A_{S}}{A_{B}}\right]$

Subsequently, the revised Fig. 6 with the corrected Eq. (5) is shown below. The typing mistake does not affect any of the results or conclusions presented in the article.

\section{References}

Västilä K, Järvelä J (2014) Modeling the flow resistance of woody vegetation using physically-based properties of the foliage and stem. Water Resour Res 50(1):229-245. https://doi.org/10.1002/2013WR013819.

The online version of the original article can be found at https://dx.doi. org/10.1007/s11368-017-1776-3

Kaisa Västilä

kaisa.vastila@aalto.fi

1 Department of Built Environment, Aalto University School of Engineering, Espoo, Finland 
Fig. 6 Usage of the flow resistance parameterization (Eqs. 2-6) in plant-scale, plant standscale, and reach-scale analyses at different relative submergences $(h / H)$. The recommended characteristic approach velocities $\left(u_{C}\right)$ are shown, with $u_{v}$ denoting the mean velocity in the vegetated part of the cross-section.

Equations are written for the drag force $(F)$, vegetative friction factor ( $\left.f^{\prime \prime}\right)$, drag-density parameter $\left(C_{D} a\right)$, drag-area parameter $\left(C_{D} a H\right)$ and vegetative Manning coefficient $\left(n_{\text {veg }}\right)$ using the one-sided leaf area $\left(A_{L}\right)$, frontal projected stem area $\left(A_{S}\right)$, unit bed area $\left(A_{B}\right)$, the leaf area per unit volume $\left(a_{L}\right)$ and the stem area per unit volume $\left(a_{S}\right)$. Values of $\chi_{F}, \chi_{S}, C_{D \chi, F}, C_{D \chi, S}, u_{\chi, F}$, and $u_{\chi, S}$ are reported in Table 2 for common riparian species. Note that all vegetative reference areas refer to the wet parts of the plants

a) Plant scale

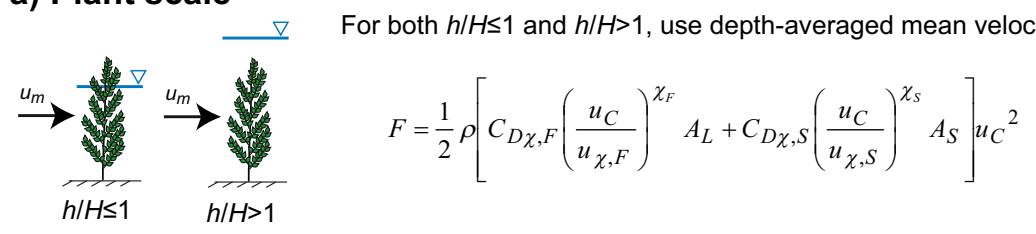

b) Plant stand scale

(fully developed flow)

$h / H \leq 1$

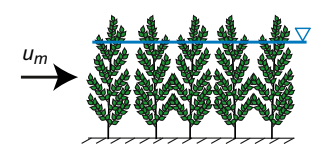

For $h / H \leq 1$, use mean velocity as $u_{C}$ with:

$f^{\prime \prime}=4\left[C_{D \chi, F}\left(\frac{u_{C}}{u_{\chi, F}}\right)^{\chi_{F}} \frac{A_{L}}{A_{B}}+C_{D \chi, S}\left(\frac{u_{C}}{u_{\chi, S}}\right)^{\chi_{S}} \frac{A_{S}}{A_{B}}\right]$

(Eq. 5)

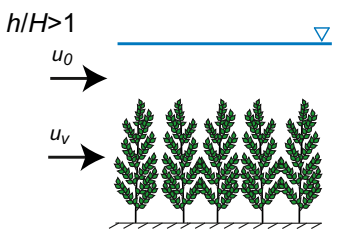

For $h / H>1$, use $u_{C}=u_{V}$ and replace the $\mathrm{C}_{\mathrm{D}} \mathrm{aH}$ parameter with:

$$
C_{D} a H=C_{D \chi, F}\left(\frac{u_{C}}{u_{\chi, F}}\right)^{\chi_{F}} \frac{A_{L}}{A_{B}}+C_{D \chi, S}\left(\frac{u_{C}}{u_{\chi, S}}\right)^{\chi_{S}} \frac{A_{S}}{A_{B}}
$$

c) Reach scale

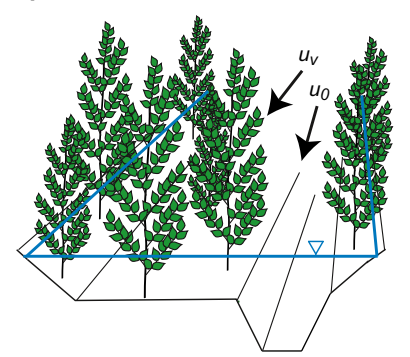

Use $u_{C}=u_{V}$ and replace the $C_{D}$ a parameter in analytical or numerical models with:

$C_{D} a=C_{D \chi, F}\left(\frac{u_{C}}{u_{\chi, F}}\right)^{\chi_{F}} a_{L}+C_{D \chi, S}\left(\frac{u_{C}}{u_{\chi, S}}\right)^{\chi_{S}} a_{S}$

$n_{\text {veg }}=\frac{K h^{1 / 6}}{\sqrt{2 g}} \sqrt{C_{D \chi, F}\left(\frac{u_{C}}{u_{\chi, F}}\right)^{\chi_{F}} \frac{A_{L}}{A_{B}}+C_{D \chi, S}\left(\frac{u_{C}}{u_{\chi, S}}\right)^{\chi_{S}} \frac{A_{S}}{A_{B}}}$ (Eq. 6) 